\title{
JORDAN ALGEBRAS WITH INTERCONNECTED IDEMPOTENTS
}

\section{KEVIN MCCRIMMON ${ }^{1}$}

Throughout this paper we will be concerned with Jordan algebras (possibly infinite-dimensional) over a field $\Phi$ of characteristic $\neq 2$ (though the results actually hold for Jordan rings as defined in [3]). In [1], N. Jacobson introduced a relation of connectedness between orthogonal idempotents in a Jordan algebra. According to this definition, two orthogonal idempotents $e_{1}$ and $e_{2}$ are connected if there is an element $x$ in the Peirce space $\mathfrak{A}_{12}$ which is invertible in the Peirce subalgebra $\mathfrak{A}_{1}\left(e_{1}+e_{2}\right)=\mathfrak{A}_{11}+\mathfrak{U}_{12}+\mathfrak{A}_{22}$ of the algebra $\mathfrak{A}$. If $y \in \mathfrak{A}_{12}$ is the inverse of $x$, then $x \cdot y=e_{1}+e_{2}$, so

$$
e_{1}=U_{e_{1}}(x \cdot y), \quad e_{2}=U_{e_{2}}(x \cdot y) \quad\left(x, y \in \mathfrak{A}_{12}\right) .
$$

An algebra is $n$-connected if it contains $n$ supplementary orthogonal idempotents $e_{1}, \cdots, e_{n}$ which are pairwise connected. The fundamental Coordinatization Theorem [1] says that an $n$-connected algebra for $n \geqq 3$ is a Jordan matrix algebra $\mathfrak{S}_{(}\left(\mathfrak{D}_{n}, \gamma\right)$, where $\mathfrak{D}$ is always alternative and is associative if $n \geqq 4$. In particular, all $n$-connected algebras for $n \geqq 4$ are special.

A notion of connectedness for associative algebras $\mathfrak{D}$ was introduced by W. Martindale in [5]: two orthogonal idempotents $e_{1}, e_{2}$ are (associative) interconnected if the (associative) Peirce spaces satisfy $\mathfrak{D}_{11}=\mathfrak{D}_{12} \mathfrak{D}_{21}, \mathfrak{D}_{22}=\mathfrak{D}_{21} \mathfrak{D}_{12}$. Then in the Jordan algebra $\mathfrak{A}=\mathfrak{D}^{+}$the Peirce space $\mathfrak{A}_{12}=\mathfrak{D}_{12}+\mathfrak{D}_{21}$ satisfies

$$
\mathfrak{A}_{11}=e_{1} \mathfrak{A}_{12}^{2} e_{1}, \quad \mathfrak{A}_{22}=e_{2} \mathfrak{A}_{12}^{2} e_{2} .
$$

This leads us to say that two orthogonal idempotents $e_{1}, e_{2}$ in an arbitrary Jordan algebra $\mathfrak{A}$ are (Jordan) interconnected if

$$
\mathfrak{A}_{11}=U_{e_{1}} \mathfrak{A}_{12}^{2}, \quad \mathfrak{A}_{22}=U_{e_{2}} \mathfrak{A}_{12}^{2} .
$$

It is actually enough if

$$
e_{1} \in U_{e_{1}} \mathfrak{P}_{12}^{2}, \quad e_{2} \in U_{e_{2}} \mathfrak{P}_{12}^{2}
$$

since, if $e_{1}=U_{e_{1}}\left(\Sigma x_{i}^{2}\right)$ for $x_{i} \in \mathfrak{Y}_{12}$, then any $a_{1} \in \mathfrak{H}_{11}$ can be expressed as $a_{1}=a_{1} \cdot e_{1}=U_{e_{1}} \sum a_{1} \cdot x_{i}^{2}=2 U_{e_{1}} \sum\left(a_{1} \cdot x_{i}\right) \cdot x_{i} \in U_{e_{1}} \mathfrak{A}_{12}^{2} \quad$ (here we use

Received by the editors August 2, 1967.

1 This research was partially supported by AFOSR Grant No. 335-63. 
$U_{e_{1}}\left(2 x_{i} \cdot\left(x_{i}^{2} \cdot a_{1}\right)-x_{i} \cdot a_{1}\right)=U_{e_{1}} U_{x_{i}} a_{1} \in U_{e_{1}} U_{\mathfrak{Q}_{12}} a_{1} \subset U_{e_{1}} \mathfrak{I}_{22}=0$ by $[3$, p. 1074]). Clearly, interconnectivity is a weaker condition than connectivity.

A supplementary family of orthogonal idempotents $e_{1}, \cdots, e_{n}$ is interconnected if the idempotents are pairwise interconnected. This is equivalent to the condition

$$
\mathfrak{A}_{i i}=P_{i i}\left(\mathfrak{A}_{i j}^{2}\right) \quad(i \neq j)
$$

on the Peirce spaces, where $P_{i i}=U_{e_{i}}$ is the projection on the Peirce space $\mathfrak{A}_{i i}$. In dealing with algebras without identity elements it is more convenient to work directly with the Peirce decompositions. If $e_{1}, \cdots, e_{n-1}$ are nonzero nonsupplementary orthogonal idempotents we have the Peirce decomposition $\mathfrak{U}=\sum_{i, j=0}^{n-1} \mathfrak{A}_{i j}$. We say the decomposition has length $n$, and tacitly assume $n \geqq 2$ (so there is at least one nonzero idempotent). Such a decomposition is interconnected if we have the condition (I) for all $i, j(0 \leqq i, j \leqq n-1)$ and also the nondegeneracy condition

$$
a_{i j} \cdot \mathfrak{A}_{j k}=0 \Rightarrow a_{i j}=0 \quad(k \neq i, j) .
$$

This is trivially satisfied if $e_{1}$ exists since as before $a_{i j}=a_{i j} \cdot e_{j} \in a_{i j}$ - $P_{j j}\left(\mathfrak{H}_{j k}^{2}\right)=P_{i j}\left\{a_{i j} \cdot \mathfrak{A}_{j k}^{2}\right\}=P_{i j}\left\{\left(a_{i j} \cdot \mathfrak{A}_{j k}\right) \cdot \mathfrak{A}_{j k}\right\}=0$ (using $P_{i j} U_{\mathfrak{I}_{j k}} \mathfrak{A}_{i j}=0$ if $k \neq i, j$ ). In particular (II) is a consequence of (I) if $\mathfrak{Q}$ has an identity; in this case the decomposition relative to $e_{1}, \cdots, e_{n-1}$ is interconnected if and only if $e_{0}, e_{1}, \cdots, e_{n-1}$ is an interconnected family of nonzero supplementary orthogonal idempotents for $e_{0}=1$ $-\left(e_{1}+\cdots+e_{n-1}\right)$. Also, for algebras without identities we need only assume

$$
\begin{aligned}
& a_{00} \cdot \mathfrak{A}_{0 k}=0 \Rightarrow a_{00}=0 \\
& a_{i 0} \cdot \mathfrak{A}_{0 k}=0 \Rightarrow a_{i 0}=0
\end{aligned} \quad(0, i, k \neq) .
$$

As a consequence of $(\mathrm{I})$ we also have

$$
\begin{aligned}
& \mathfrak{U}_{i k}=\mathfrak{A}_{i j} \mathfrak{A}_{j k} \quad(i, j, k \neq) \\
& \mathfrak{A}_{i k}=\mathfrak{U}_{i j}\left(\mathfrak{A}_{i j} \mathfrak{A}_{i k}\right) \quad(i, j, k \neq) .
\end{aligned}
$$

These two are equivalent since the first implies $\mathfrak{A}_{i j} \mathfrak{A}_{j k}=\mathfrak{A}_{i j}\left(\mathfrak{A}_{j i \mathfrak{i}} \mathfrak{I}_{i k}\right)$ and since $\mathfrak{A}_{i j} \mathfrak{A}_{i k} \subset \mathfrak{A}_{j k}$. We will derive the first one, and by symmetry we may assume $i \neq 0$. The calculation is the one we have used several times:

$$
\begin{aligned}
\mathfrak{A}_{i k} & =\mathfrak{A}_{i k} \cdot e_{i} \in \mathfrak{U}_{i k} \cdot P_{i i}\left(\mathfrak{U}_{i j}^{2}\right) \quad(\text { by }(\mathrm{I})) \\
& =P_{i k}\left\{\mathfrak{I}_{i k} \cdot \mathfrak{H}_{i j}^{2}\right\}=P_{i k}\left\{\left(\mathfrak{U}_{i k} \cdot \mathfrak{A}_{i j}\right) \cdot \mathfrak{A}_{i j}\right\} \subset \mathfrak{A}_{j k} \cdot \mathfrak{A}_{i j} .
\end{aligned}
$$


An algebra is $n$-interconnected if it has an interconnected Peirce decomposition of length $n$.

If $\mathfrak{A}$ is $n$-interconnected, it is $m$-interconnected for any $m \leqq n$. Indeed, unlike connectedness, interconnectedness is preserved if certain of the idempotents are "lumped together." For example, in a Jordan matrix algebra the diagonal idempotents $e_{i i}$ are connected, but $e_{11}$ and $e_{22}+e_{33}$ are interconnected without being connected. For algebras with identities we have the further properties that a homomorphic image of an $n$-interconnected algebra is $n$-interconnected (or zero), that $\mathfrak{B}$ is $n$-interconnected if it has an $n$-interconnected subalgebra $\mathfrak{A}$ with the same identity element, and hence that the split null extension $\mathbb{E}=\mathfrak{A} \oplus \mathfrak{M}$ of an $n$-interconnected algebra $\mathfrak{A}$ by a unital bimodule $\mathfrak{M}$ is $n$-interconnected. These don't seem to hold for algebras without identities. Note that if we adjoin an identity to $\mathfrak{P}$, the resulting algebra is never interconnected.

One reason that interconnectedness is interesting is

Theorem 1. Any Peirce decomposition of an arbitrary simple Jordan algebra is interconnected.

There is no reason to believe the decomposition must actually be connected even if the idempotents are primitive, though this is true for finite-dimensional algebras or algebras satisfying the axioms of $[2$, p. 246]. The consideration of algebras without identities is necessary here because a simple Jordan algebra need not contain an identity in general.

The main result of this paper will be to show that, in analogy with the case of connected idempotents,

Theorem 2. Any n-interconnected Jordan algebra for $n \geqq 4$ is special.

This result was first conjectured by N. Jacobson. As a corollary of Theorems 1 and 2 we have

THEOREM 3. Any simple Jordan algebra with three nonzero orthogonal idempotents whose sum is not 1 is special.

1. Proof of Theorem 1. The main tool we will use in this section is the

Lemma [4, p. 189]. If $e$ is an idempotent in a Jordan algebra $\mathfrak{A}$, $\mathfrak{B}_{1 / 2}$ a subspace of the Peirce space $\mathfrak{H}_{1 / 2}=\mathfrak{H}_{1 / 2}(e)$ such that $\left(\mathfrak{A}_{0}+\mathfrak{F}_{1}\right) \mathfrak{B}_{1 / 2}$ $\subset \mathfrak{B}_{1 / 2}$, then $\mathfrak{B}_{i}=P_{i}\left\{\mathfrak{A}_{1 / 2} \mathfrak{B}_{1 / 2}\right\}$ is an ideal in $\mathfrak{A}_{i}$ for $i=0,1$.

This will allow us to construct ideals: to verify that $\mathfrak{B}=\mathfrak{B}_{0}+\mathfrak{B}_{1 / 2}+\mathfrak{B}_{1}$ is an ideal we need only verify $\left(\mathfrak{B}_{0}+\mathfrak{B}_{1}\right) \mathfrak{A}_{1 / 2} \subset \mathfrak{B}_{1 / 2}$. In particular, with $\mathfrak{B}_{1 / 2}=\mathfrak{A}_{1 / 2}$ we see $\mathfrak{B}=P_{0}\left\{\mathfrak{A}_{1 / 2}\right\}+\mathfrak{A}_{1 / 2}+P_{1}\left\{\mathfrak{A}_{1 / 2}^{2}\right\}$ is always an ideal. 
Let $\mathfrak{A}=\sum_{i, j=0}^{n=1} \mathfrak{A}_{i j}$ be the Peirce decomposition of a simple Jordan algebra $\mathfrak{A}$ relative to nonzero orthogonal idempotents $e_{1}, \cdots, e_{n-1}$. The first step will be to establish (I). Applying the Lemma with $e=e_{j}, \mathfrak{B}_{1 / 2}=\mathfrak{A}_{1 / 2}=\sum_{i \neq j} \mathfrak{A}_{i j}$ we see $\mathfrak{B}=\mathfrak{B}_{0}+\mathfrak{B}_{1 / 2}+\mathfrak{B}_{1}$ is an ideal; it is nonzero since $\mathfrak{A}_{1 / 2} \neq 0$, so by simplicity it must be all of $\mathfrak{A}$. Then $\mathfrak{A}_{i i}=P_{i i}\left(\mathfrak{U}_{1 / 2}^{2}\right)=P_{i i}\left(\mathfrak{H}_{i j}^{2}\right)$ for $i \neq j$. This gives us (I) as long as $j \neq 0$; for $j=0$ we do the same thing with $e=e_{1}+\cdots+e_{n-1}, \mathfrak{B}_{1 / 2}=\sum_{i \neq 0} \mathfrak{A}_{i 0}$, to get $\mathfrak{A}_{i i}=P_{i i}\left(\mathfrak{H}_{i 0}^{2}\right)$ (in order to have $\mathfrak{B} \neq 0$ we must use the hypothesis that $e_{1}, \cdots, e_{n-1}$ are not supplementary, i.e., $e \neq 1$ ).

To finish showing that our Peirce decomposition is connected we we must establish $\left(\mathrm{II}_{0}\right)$. First suppose $a_{00} \cdot \mathfrak{A}_{0 k}=0$. Then $a_{00} \cdot \mathfrak{H}_{0 j}$ $=a_{00}\left(\mathfrak{A}_{0 k} \mathfrak{H}_{k j}\right)=\left(a_{00} \mathfrak{A}_{0 k}\right) \mathfrak{A}_{k j}=0$ for any $j \neq k$ by (III). Letting $e$ $=e_{1}+\cdots+e_{n-1}$ we see $a_{00} \in \mathfrak{B}=\left\{b_{0} \in \mathfrak{A}_{0}(e)=\mathfrak{A}_{00} \mid b_{0} \mathfrak{A}_{1 / 2}(e)=0\right\}$. But $\mathfrak{B}$ is an ideal in $\mathfrak{A}\left(\mathfrak{B A}_{1 / 2}=\mathfrak{B A}_{1}=0\right.$ and $\mathfrak{B A}_{0} \subset \mathfrak{B}$ since $\left(b_{0} a_{0}\right) \mathfrak{A}_{1 / 2}$ $\left.\subset b_{0}\left(a_{0} \mathfrak{A}_{1 / 2}\right)+a_{0}\left(b_{0} \mathfrak{A}_{1 / 2}\right)=0\right)$. Since $\mathfrak{B}$ isn't $\mathfrak{A}$ it must be 0 by simplicity, and $a_{00}=0$.

Now suppose $a_{0 i} \mathfrak{A}_{0 k}=0$ for $0, i, k \neq$. We will have to work a little harder to construct a suitable ideal containing $a_{0 i}$. By assumption we must have at least three distinct indices in this case. As before, $a_{0 i} \mathfrak{A}_{0 j}=a_{0 i}\left(\mathfrak{A}_{0 k} \mathfrak{A}_{k j}\right)=\left(a_{0 i} \mathfrak{U}_{0 k}\right) \mathfrak{A}_{k j}=0$ for any $j \neq 0, i$ and thus $a_{0 i} \in \mathfrak{B}_{0 i}$ $=\left\{b_{0 i} \in \mathfrak{A}_{0 i} \mid b_{0 i} \mathfrak{A}_{0 k}=0\right.$ for all $\left.k \neq 0, i\right\}$. Note also that

$$
P_{i i}\left\{\mathfrak{A}_{0 i} \mathfrak{B}_{0 i}\right\}=0, \quad \mathfrak{A}_{00} \mathfrak{B}_{0 i}=0
$$

since $P_{i i}\left\{\mathfrak{A}_{0 i} \mathfrak{B}_{0 i}\right\}=P_{i i}\left\{\left(\mathfrak{A}_{i k} \mathfrak{U}_{k 0}\right) \mathfrak{B}_{0 i}\right\}=P_{i i}\left\{\mathfrak{A}_{i k}\left(\mathfrak{A}_{k 0} \mathfrak{B}_{0 i}\right)\right\}=0$ by (III) and $\mathfrak{A}_{00} \mathfrak{B}_{0 i}=P_{00}\left(\mathfrak{H}_{0 k}^{2}\right) \mathfrak{B}_{0 i} \subset \mathfrak{A}_{0 k}\left(\mathfrak{A}_{0 k} \mathfrak{B}_{0 i}\right)=0$ by (I), where we have already established (I) and (III) for our decomposition. We also need

$$
\mathfrak{B}_{0 i} \mathfrak{A}_{i i} \subset \mathfrak{B}_{0 i}, \quad \mathfrak{B}_{0 i} \mathfrak{A}_{i j} \subset \mathfrak{B}_{0 j} \quad(0, i, j \neq)
$$

which follows from $\left(\mathfrak{B}_{0 i} \mathfrak{A}_{i i}\right) \mathfrak{A}_{0 k}=\left(\mathfrak{B}_{0 i} \mathfrak{A}_{0 k}\right) \mathfrak{A}_{i i}=0$ and $\left(\mathfrak{B}_{0 i} \mathfrak{A}_{i j}\right) \mathfrak{A}_{0 i}$ $=-\left(\mathfrak{A}_{0 i} \mathfrak{A}_{i j}\right) \mathfrak{B}_{0 i}+\left(\mathfrak{A}_{0 i} \mathfrak{B}_{0 i}\right) \mathfrak{A}_{i j} \subset \mathfrak{A}_{0 j} \mathfrak{B}_{0 i}+\mathfrak{A}_{00} \mathfrak{A}_{i j}$ (by (a)) $=0$ and the result that if $a_{0 k}$ annihilates one $\mathfrak{A}_{0 l}$ it annihilates them all. Now we can define the ideal $\mathfrak{B}$ : take $e=e_{1}+\cdots+e_{n-1}, \mathfrak{B}_{1 / 2}=\sum_{i \neq 0} \mathfrak{B}_{0 i}$ $C \sum_{i \neq 0} \mathfrak{A}_{0 i}=\mathfrak{A}_{1 / 2}$, and let $\mathfrak{B}=\mathfrak{B}_{0}+\mathfrak{B}_{1 / 2}+\mathfrak{B}_{1}$ where $\mathfrak{B}_{i}=P_{i}\left\{\mathfrak{A}_{1 / 2} \mathfrak{B}_{1 / 2}\right\}$ for $i=0,1$. Noticing that $\mathfrak{B}_{1}=0$ since $\mathfrak{A}_{1}=\sum_{i, 0 \neq \mathfrak{A}_{i i}}+\sum_{i, j, 0 \neq} \mathfrak{A}_{i j}$ and $P_{i i}\left\{\mathfrak{A}_{1 / 2} \mathfrak{B}_{1 / 2}\right\}=P_{i i}\left\{\mathfrak{A}_{i 0} \mathfrak{B}_{i 0}\right\}=0$ by (a), $P_{i j}\left\{\mathfrak{A}_{1 / 2} \mathfrak{B}_{1 / 2}\right\}=\mathfrak{A}_{0 i} \mathfrak{B}_{0 j}+\mathfrak{A}_{0 j} \mathfrak{B}_{0 i}$ $=0$ by definition of $\mathfrak{B}_{0 k}$, the Lemma shows we have only to verify $\left(\mathfrak{A}_{0}+\mathfrak{A}_{1}\right) \mathfrak{B}_{1 / 2} \subset \mathfrak{B}_{1 / 2}$ and $\mathfrak{B}_{0} \mathfrak{A}_{1 / 2} \subset \mathfrak{B}_{1 / 2}$. But $\mathfrak{A}_{0} \mathfrak{B}_{1 / 2}=\mathfrak{A}_{00} \sum \mathfrak{B}_{0 i}=0$ by (a),

$$
\begin{aligned}
\mathfrak{A}_{1} \mathfrak{B}_{1 / 2}= & \left(\sum \mathfrak{A}_{i i}+\sum \mathfrak{A}_{i j}\right) \mathfrak{B}_{1 / 2}=\sum \mathfrak{A}_{i i} \mathfrak{B}_{0 i} \\
& +\sum \mathfrak{A}_{i j}\left(\mathfrak{B}_{0 i}+\mathfrak{B}_{0 j}\right) \subset \mathfrak{B}_{1 / 2}
\end{aligned}
$$

by (b), and $\mathfrak{B}_{0} \mathfrak{A}_{1 / 2}=\left\{\sum P_{00}\left(\mathfrak{H}_{0 i} \mathfrak{B}_{0 i}\right)\right\} \mathfrak{A}_{1 / 2}$, where 


$$
P_{00}\left(\mathfrak{A}_{0 i} \mathfrak{B}_{0 i}\right) \mathfrak{A}_{0 j}=\mathfrak{A}_{0 i}\left(\mathfrak{B}_{0 i} \mathfrak{A}_{0 j}\right)+\mathfrak{B}_{0 i}\left(\mathfrak{A}_{0 i} \mathfrak{A}_{0 j}\right) \subset 0+\mathfrak{B}_{0 i} \mathfrak{A}_{i j} \subset \mathfrak{B}_{0 j}
$$

by (b) if $j \neq i$ and

$$
\begin{aligned}
P_{00}\left(\mathfrak{A}_{0 i} \mathfrak{B}_{0 i}\right) \mathfrak{A}_{0 i} & =P_{00}\left(\mathfrak{A}_{0 i} \mathfrak{B}_{0 i}\right)\left(\mathfrak{A}_{0 j} \mathfrak{A}_{i j}\right) \\
& =\left\{P_{00}\left(\mathfrak{A}_{0 i} \mathfrak{B}_{0 i}\right) \mathfrak{A}_{0 j}\right\} \mathfrak{A}_{i j} \subset \mathfrak{B}_{0 j} \mathfrak{A}_{i j} \subset \mathfrak{B}_{0 i}
\end{aligned}
$$

by (III), (b), and the previous case (we can find $j \neq 0, i$ since there are at least three indices). Thus $\mathfrak{B}$ is an ideal; clearly $\mathfrak{B} \neq \mathfrak{A}$, so $\mathfrak{B}=0$, $a_{0 i}=0$, and $\left(\mathrm{II}_{0}\right)$ is established.

This completes the proof that any Peirce decomposition of a simple Jordan algebra is interconnected in the sense that it satisfies (I) and (II).

2. A criterion. For the proof of Theorem 2 we will need a result which allows us to reconstruct an associative algebra $\mathfrak{D}$ from a Jordan algebra $\mathfrak{A}$ and a specialization of $\mathfrak{A}$ in $\mathfrak{D}$, that is, a homomorphism $\mathfrak{Y} \rightarrow \mathfrak{D}^{+}$of Jordan algebras. If $\left\{e_{i}\right\}$ are nonzero orthogonal idempotents in $\mathcal{A}$, then their images $\left\{\delta\left(e_{i}\right)\right\}$ under a specialization $\delta$ are orthogonal idempotents in $\mathfrak{D}$ (which must be nonzero if the $\left\{e_{i}\right\}$ are interconnected and $\delta \neq 0$ ).

CRITERION. Let $\delta$ be a specialization of a Jordan algebra $\mathfrak{A}$ in an associative algebra $\mathfrak{D}$ such that the image $\delta(\mathfrak{H})$ generates $\mathfrak{D}$. If $\mathfrak{U}=\sum_{i, j=0}^{n-1} \mathfrak{A}_{i j}$ is an interconnected Peirce decomposition relative to $e_{1}, \cdots, e_{n-1}$ for $n \geqq 3$ and $\mathfrak{D}=\sum_{i, j=0}^{n-1} \mathfrak{D}_{i j}$ the corresponding Peirce decomposition relative to $\delta\left(e_{1}\right), \cdots, \delta\left(e_{n-1}\right)$, then the Peirce spaces satisfy

$$
\mathfrak{D}_{i j}=\delta_{i j}\left(\mathfrak{A}_{i j}\right), \quad \mathfrak{D}_{i i}=\delta_{i i j}\left(\mathfrak{A}_{i j} \otimes \mathfrak{A}_{i j}\right) \quad(i \neq j),
$$

where if $P_{i j}: \mathfrak{D} \rightarrow \mathfrak{D}_{i j}$ are the Peirce projections in $\mathfrak{D}$ the maps $\delta_{i j}: \mathfrak{A}_{i j} \rightarrow \mathfrak{I}_{i j}$ and $\delta_{i i j}: \mathfrak{A}_{i j} \otimes \mathfrak{A}_{i j} \rightarrow \mathfrak{D}_{i i}$ are defined by

$$
\begin{aligned}
\delta_{i j}(a) & =P_{i j} \delta(a) \quad\left(a \in \mathfrak{A}_{i j}\right) \\
\delta_{i i j}(a \otimes b) & =\delta_{i j}(a) \delta_{j i}(b)=P_{i i} \delta(a) \delta(b) \quad\left(a, b \in \mathfrak{A}_{i j}\right)
\end{aligned}
$$

and where multiplication in $\mathfrak{D}$ is completely determined for $i, j, k \neq$ by the relations

$$
\begin{array}{rlrl}
\delta_{i j}(a) \delta_{j k}(b) & =2 \delta_{i k}(a \cdot b) \quad\left(a \in \mathfrak{A}_{i j}, b \in \mathfrak{A}_{j k}\right) \\
\delta_{i i j}(a \otimes b) \delta_{i k}(c) & =4 \delta_{i k}(a \cdot(b \cdot c)) & & \left(a, b \in \mathfrak{A}_{i j}, c \in \mathfrak{A}_{i k}\right) \\
\delta_{i j}(a) \delta_{j j k}(b \otimes c) & =4 \delta_{i j}((a \cdot b) \cdot c) & & \left(a \in \mathfrak{A}_{i j}, b, c \in \mathfrak{A}_{j k}\right) \\
\delta_{i i j}(a \otimes b) \delta_{i i k}(c \otimes d) & =4 \delta_{i i j}(a \otimes((b \cdot c) \cdot d)) \\
& =4 \delta_{i i k}((a \cdot(b \cdot c)) \otimes d) \\
& \left(a, b \in \mathfrak{A}_{i j}, c, d \in \mathfrak{A}_{i k}\right) .
\end{array}
$$


Proof. (1.2) and (1.3) are used to define the linear maps $\delta_{i j}$ and $\delta_{i i j}$; note that $\delta\left(\mathfrak{A}_{i j}\right) \subset \mathfrak{D}_{i j}+\mathfrak{D}_{j i}$ for all $i, j$. Then (1.4) follows from $2 \delta_{i k}(a \cdot b)=2 P_{i k} \delta(a \cdot b)=P_{i k}\{\delta(a) \delta(b)+\delta(b) \delta(a)\}=\delta_{i j}(a) \delta_{j k}(b)$ by $\delta(a)$ $=\delta_{i j}(a)+\delta_{j i}(a), \delta(b)=\delta_{j k}(b)+\delta_{k j}(b)$ and the Peirce orthogonality relations. From this (1.5)-(1.7) follow by using the definition (1.3). Also (1.7) and the relation (IV) show that the image of $\delta_{i i 4}$ does not depend on the index $j$. The formulas (1.3)-(1.7) show that the images of the $\delta_{i j}$ and $\delta_{i i j}$ span a subalgebra of $\mathfrak{D}$ (we need $n \geqq 3$ to be able to find $i, j, k \neq)$. Since $\delta\left(\mathfrak{A}_{i j}\right) \subset \delta_{i j}\left(\mathfrak{A}_{i j}\right)+\delta_{j i}\left(\mathfrak{A}_{i j}\right)$ and

$$
\delta\left(\mathfrak{A}_{i i}\right)=\delta\left(P_{i i}\left(\mathfrak{A}_{i j}^{2}\right)\right) \subset P_{i i} \delta\left(\mathfrak{A}_{i j}\right) \delta\left(\mathfrak{A}_{i j}\right)=\delta_{i i j}\left(\mathfrak{A}_{i j} \otimes \mathfrak{A}_{i j}\right)
$$

by interconnectivity (I) this subalgebra contains the generating set $\delta(\mathfrak{U})$, and hence must be all of $\mathfrak{D}$. This establishes (1.1).

At this point we digress to obtain two theorems about special universal envelopes. It seems necessary to require the existence of identity elements.

THEOREM 4. If $\delta$ is a specialization of an n-interconnected Jordan algebra $\mathfrak{A}$ with identity in an associative algebra $\mathfrak{D}$ for $n \geqq 3$ such that

(2.1) $\delta(\mathfrak{A})$ generates $\mathfrak{D}$,

(2.2) $\delta_{i j}(a)=P_{i j}(a)=0$ implies $a=0$ if $a$ is in the Peirce space

$\mathfrak{A}_{i j}(i \neq j)$ relative to the interconnected family $\left\{e_{k}\right\}$

then $\mathfrak{A}$ is a special Jordan algebra and $(\mathfrak{D}, \delta)$ is a special universal envelope for $\mathfrak{A}$.

Proof. We first show $\delta$ is $1-1$, so $\mathfrak{A}$ is special. If $\mathfrak{U}=\sum \mathfrak{A}_{i j}$ is the Peirce decomposition relative to the idempotents $\left\{e_{i}\right\}$, then the kernel has the form $\Re=\sum \Omega_{i j}$ for $\Re_{i j}=\Re \cap \mathfrak{A}_{i j}$. (2.2) implies $\Omega_{i j}=0$ for $i \neq j$, so $\Omega_{i i} \mathfrak{A}_{i j} \subset \Omega_{i j}=0$, and by interconnectivity (II) we have $\Omega_{i i}=0$ too. Thus $\Re=0$ and $\delta$ is $1-1$. Note that we didn't need the existence of an identity here.

To prove $(\mathfrak{D}, \delta)$ is a universal envelope we must demonstrate the universal property that any specialization $\gamma$ of $\mathfrak{A}$ in an associative algebra $\mathbb{E}$ factors uniquely through $\delta$, i.e., there is a homomorphism $\phi$ of associative algebras making

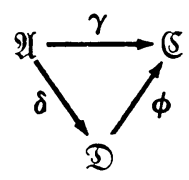

commutative. Uniqueness is equivalent to the fact that $\delta(\mathfrak{I})$ generates $\mathfrak{I}$, hence follows from (2.1). 
For the existence of $\phi$, it suffices to assume $\gamma(\mathfrak{H})$ generates $\mathfrak{C}$. Then the Criterion is applicable to $\delta$ and $\gamma$, giving us maps $\delta_{i j}$, $\delta_{i i j}$ and $\gamma_{i j}, \gamma_{i i j}$. Since multiplication in $\mathbb{I}$ and $\mathbb{S}$ is completely determined by $(1.1)-(1.7)$, the map $\phi$ defined by $\phi\left(\delta_{i j}(a)\right)=\gamma_{i j}(a), \phi\left(\delta_{i i j}(a \otimes b)\right)$ $=\gamma_{i i j}(a \otimes b)$ for $a, b \in \mathfrak{A}_{i j}$ will be a homomorphism if it is well defined. Consequently, we need only verify

$\delta_{i j}(a)=0 \Rightarrow \gamma_{i j}(a)=0 \quad\left(a \in \mathfrak{A}_{i j}\right)$

$\delta_{i i j}(p)=\delta_{i i k}(q) \Rightarrow \gamma_{i i j}(p)=\gamma_{i i k}(q) \quad\left(p \in \mathfrak{A}_{i j} \otimes \mathfrak{A}_{i j}, q \in \mathfrak{A}_{i k} \otimes \mathfrak{A}_{i k}\right)$.

The first of these follows trivially from (2.2). For the second, if $\delta_{i i j}(p)=\delta_{i i k}(q)$ for $p=\sum a_{r} \otimes b_{r}, q=\sum c_{s} \otimes d_{s}\left(a_{r}, b_{r} \in \mathfrak{A}_{i j}, c_{s}, d_{s} \in \mathfrak{A}_{i k}\right)$, then multiplying on the right by $\delta_{i k}(x)=\delta_{i j}(y) \delta_{j k}(z)$ for

$$
x=2 y \cdot z \quad\left(x \in \mathfrak{A}_{i k}, y \in \mathfrak{A}_{i j}, z \in \mathfrak{A}_{j k}\right)
$$

yields $4 \delta_{i k}\left(\sum a_{r} \cdot\left(b_{r} \cdot x\right)\right)=4 \delta_{i k}\left(\sum\left\{c_{s} \cdot\left(d_{s} \cdot y\right)\right\} \cdot z\right)$, by (1.5), (1.4). From (2.2) we see $\sum a_{r} \cdot\left(b_{r} \cdot x\right)=\sum\left\{c_{s} \cdot\left(d_{s} \cdot y\right)\right\} \cdot z$. Retracing our steps with $\delta$ 's replaced by $\gamma$ 's shows $\gamma_{i i j}(p)-\gamma_{i i k}(q)=c_{i i} \in \mho_{i i}$ is killed on the right by $\gamma_{i k}(x)$. By interconnectivity (III) such $x$ span $\mathfrak{A}_{i k}$, so $c_{i \imath} \gamma_{i k}\left(\mathfrak{A}_{i k}\right)=0$ and

$$
c_{i i} \mathfrak{E}_{i i}=c_{i i} \gamma_{i i k}\left(\mathfrak{H}_{i k} \otimes \mathfrak{H}_{i k}\right)=c_{i i} \gamma_{i k}\left(\mathfrak{A}_{i k}\right) \gamma_{k i}\left(\mathfrak{H}_{i k}\right)=0
$$

by the Criterion. But now $\gamma\left(e_{i}\right) \in \mathfrak{C}_{i i}$ (here's where we need the existence of an identity, to guarantee there is an $\left.e_{i}\right)$, so $c_{i i}=0$ and $\gamma_{i i j}(p)$ $=\gamma_{i i k}(q)$ as required.

A particular case gives one version of

Martindale's THeOREM [5]. If $\mathfrak{D}$ is an associative algebra with involution * over a field of characteristic not two with $1=e_{1}+\cdots+e_{n}$ the sum of $n \geqq 3$ interconnected orthogonal idempotents which are

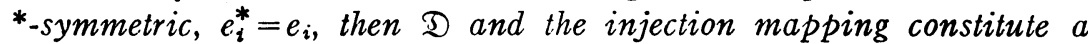
special universal envelope for the Jordan algebra $\mathfrak{A}=\mathfrak{E}\left(\mathfrak{D},{ }^{*}\right)$ of symmetric elements.

Proof. We claim the injection $\delta: \mathfrak{A} \rightarrow \mathfrak{D}$ satisfies the conditions of Theorem 4. The Peirce spaces of $\mathfrak{D}$ satisfy $\mathfrak{D}_{i j}^{*}=\mathfrak{D}_{j i}$, so the Peirce spaces of $\mathfrak{A}$ are $\mathfrak{A}_{i j}=\left\{d+d^{*} \mid d \in \mathfrak{D}_{i j}\right\}$ (here we need characteristic $\neq 2$ ). For $i \neq j$ thisimmediately establishes (2.2) and shows $\mathfrak{D}_{i j}=e_{i} \mathfrak{A}_{i j} e_{j}$ is generated by $\delta(\mathfrak{R})$; since $\mathfrak{D}_{i i}=\mathfrak{D}_{j i} \mathfrak{D}_{i j}$ by associative-interconnectivity we see $\delta(\mathfrak{H})$ generates all of $\mathfrak{D}$, and (2.1) holds. All that is left to verify is that the $e_{i}$ are Jordan-interconnected in $\mathfrak{A}$. If $\mathfrak{D}_{i i}=\mathfrak{D}_{i j} \mathfrak{D}_{j i}$ is spanned by all $d_{i i}=d_{i j} d_{j i}$ then $\mathfrak{A}_{i i}$ is spanned by all

$$
d_{i i}+d_{i i}^{*}=d_{i j} d_{j i}+d_{j i}^{*} l_{i j}^{*}=2 P_{i i}\left\{\left(d_{i j}+d_{i j}^{*}\right) \cdot\left(d_{j i}+d_{j i}^{*}\right)\right\} \in P_{i i}\left(\mathfrak{A}_{i j}^{2}\right)
$$

by orthogonality and $d_{i j}^{*} \in I_{j i}, d_{j i}^{*} \in \mathfrak{I}_{i j}$. 
3. Proof of Theorem 2. Let $\mathfrak{A}=\sum_{i, j=0}^{n-1} \mathfrak{A}_{i j}$ be an interconnected Peirce decomposition of the Jordan algebra $\mathfrak{A}$ relative to $e_{1}, \cdots, e_{n-1}$ where $n \geqq 4$. To prove that $\mathfrak{A}$ is special we shall construct an associative algebra $\mathfrak{D}$ and an imbedding $\delta: \mathfrak{A} \rightarrow \mathfrak{T}^{+}$.

Recall that if $e$ is any idempotent, then the map $a \rightarrow 2 L_{a}$ defines the Peirce specialization of the Peirce subalgebra $\mathfrak{H}_{0}(e)$ (or $\mathfrak{A}_{1}(e)$ ) in the associative algebra of linear transformations on $\mathfrak{H}_{1 / 2}(e)\left(2 L_{a}^{2}=L_{a^{2}}\right.$ on $\mathfrak{A}_{1 / 2}$, since $U_{a}=2 L_{a}^{2}-L_{a^{2}}=0$ there [3, p. 1074]). Thus in our situation we have Peirce specializations $\gamma^{(k)}$ of the Peirce subalgebras $\mathfrak{U}^{(k)}$ $=\mathfrak{A}_{0}\left(e_{k}\right)$ (we agree that $\mathfrak{A}_{0}\left(e_{0}\right)$ is to be interpreted as $\mathfrak{A}_{1}\left(e_{1}+\cdots+e_{n-1}\right)$ if $\mathfrak{A}$ does not have an identity) in associative algebras $\mathfrak{S}^{(k)}$, the algebra of linear transformations in $\mathfrak{A}_{1 / 2}\left(e_{k}\right)$ generated by $\gamma^{(k)}\left(\mathfrak{A}^{(k)}\right)$. Each $\mathfrak{A}^{(k)}$ has the interconnected Peirce decomposition $\mathfrak{A}^{(k)}=\sum_{i, j \neq k} \mathfrak{A}_{i j}$ of length $n-1 \geqq 3$; since $\gamma^{(k)}\left(\mathfrak{A}^{(k)}\right)$ generates $\mathfrak{S}^{(k)}$ by definition we may apply the Criterion to conclude that $\mathbb{S}^{(k)}$ has the Peirce decomposition $\mathfrak{S}^{(k)}=\sum_{i, j \neq k} \mathbb{E}_{i j}^{(k)}$, where

$$
\mathfrak{E}_{i j}^{(k)}=\gamma_{i j}^{(k)}\left(\mathfrak{A}_{i j}\right), \quad \mathfrak{S}_{i i}^{(k)}=\gamma_{i i j}^{(k)}\left(\mathfrak{A}_{i j} \otimes \mathfrak{A}_{i j}\right) \quad(i \neq j) .
$$

Note also that $\boldsymbol{\gamma}^{(k)}$ satisfies (2.2), since $\boldsymbol{\gamma}_{i j}^{(\boldsymbol{k})}(a)$ for $a \in \mathfrak{A}_{i j}$ is just the restriction of $2 L_{a}$ to $\mathfrak{A}_{j k} \subset \mathfrak{A}_{1 / 2}\left(e_{k}\right)=\sum_{l \neq k} \mathfrak{A}_{l k}$, and $a_{i j} \cdot \mathfrak{A}_{j k}=0$, for $i, j, k \neq$ implies $a_{i j}=0$ by interconnectivity (II). Thus each $\gamma_{i j}^{(k)}$ is $1-1$ for $i \neq j$, and as we saw in the proof of Theorem 4 this guarantees $\gamma^{(k)}$ is injective.

The first step will be to construct an algebra $\mathfrak{D}=\sum \mathfrak{D}_{i j}$ and maps $\delta_{i j}, \delta_{i i j}$ satisfying (1.1)-(1.7). We will choose the $\mathfrak{D}_{i j}$ to be isomorphic to the spaces $\mathbb{E}_{i j}^{(\boldsymbol{k})}(k \neq i, j)$ under bijections $\phi_{i j}^{(\boldsymbol{k})}$ such that
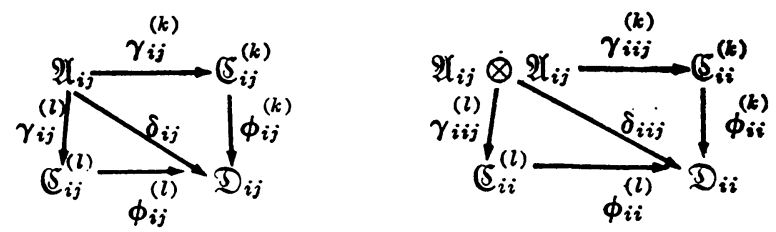

are commutative for $i, j, k, l, \neq$. We can use these to define $\delta_{i j}, \delta_{i: j}$ if we can find bijections $\phi_{i j}^{(k)}$ making the outer squares dommutative. In turn, it suffices to find bijections $\gamma_{i j}^{(k l)}$ making the upper triangles of
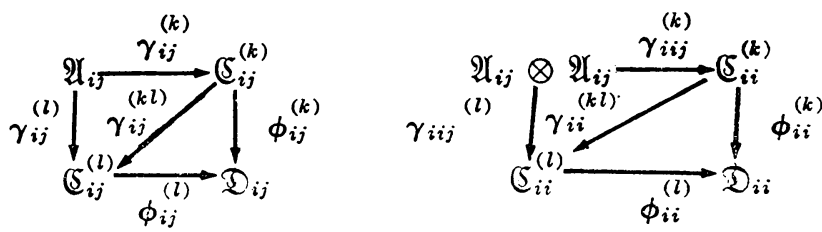
commutative, since this allows us to define $\mathfrak{I}_{i j}$ and bijections $\phi_{i j}^{(\boldsymbol{k})}$ making the whole diagrams commutative. The case of $\gamma_{i j}^{(k l)}$ for $i \neq j$ is trivial since we have seen $\gamma_{i j}^{(k)}, \gamma_{i j}^{(l)}$ are bijections. For $i=j$ we need only verify that $\gamma_{i i}^{(k l)}\left(\gamma_{i i j}^{(k)}(p)\right)=\gamma_{i i j}^{(l)}(p)$ is well defined, i.e., $\gamma_{i i j}^{(k)}(p)=0$ implies $\gamma_{i i j}^{(l)}(p)=0$, since reversing $k$ and $l$ will show $\gamma_{i i}^{(k l)}$ is bijective. If $p=\sum a_{r} \otimes b_{r}$ for $a_{r}, b_{r} \in \mathfrak{A}_{i j}$, then $\gamma_{i i j}^{(l)}(p)$ is the restriction of $4 \sum L_{a_{r}} L_{b_{r}}$ to $\mathfrak{A}_{i l}$. For $x=y \cdot z$ where $x \in \mathfrak{A}_{i l}, y \in \mathfrak{A}_{i k}, z \in \mathfrak{A}_{k l}$ we have $\left[\gamma_{i j}^{(l)}(p)\right] x$ $=4 \sum a_{r} \cdot\left(b_{r} \cdot(y \cdot z)\right)=4\left\{\sum a_{r} \cdot\left(b_{r} \cdot y\right)\right\} \cdot z=\left\{\left[\gamma_{i i j}^{(k)}(p)\right] y\right\} \cdot z$. Since such $x$ span $\mathfrak{A}_{i l}$ by (III), we see $\gamma_{i i j}^{(k)}(p)=0$ implies $\gamma_{i j}^{(l)}(p)=0$.

We next define an algebra structure on $\mathfrak{D}$ satisfying the usual Peirce relations. It suffices to define maps $\mathfrak{D}_{i j} \otimes \mathfrak{D}_{j k} \rightarrow \mathfrak{D}_{i k}(i, j, k$ not necessarily distinct). Since $n \geqq 4$ we can always find $l \neq i, j, k$, and we can use the multiplication in $\mathfrak{S}^{(l)}$ to give us $\mathfrak{D}_{i j} \otimes \mathfrak{D}_{j k} \rightarrow \mathfrak{S}_{i j}^{(l)} \otimes \mathfrak{S}_{j \mathfrak{k}}^{(l)}$ $\rightarrow \mathfrak{S}_{\mathfrak{i k}}^{(l)} \rightarrow \mathfrak{D}_{i k}$; this is independent of $l$ because

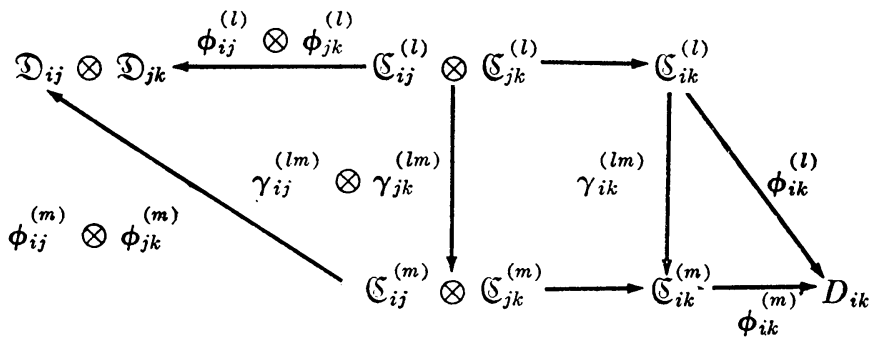

is commutative. Indeed, the two triangles are commutative by (4); the square is commutative because of (1.2)-(1.7) and the formulas $\gamma_{i j}^{(l m)}\left(\gamma_{i j}^{(l)}(p)\right)=\gamma_{i j}^{(m)}(p)$ and $\gamma_{i i}^{(l m)}\left(\gamma_{i i j}^{(l)}(p)\right)=\gamma_{i i j}^{(m)}(p)$ from (4). Note that we have (1.1)-(1.7) in $\mathfrak{D}$ by putting together (3) and (5).

If $\mathfrak{D}^{(k)}$ denotes the subalgebra $\mathfrak{D}^{(k)}=\sum_{i, j \neq k} \mathfrak{D}_{i j}$, then by construction $\phi^{(k)}=\sum \phi_{i j}^{(\boldsymbol{k})}$ is an isomorphism of $\mathfrak{C}^{(k)}$ onto $\mathfrak{D}^{(k)}$. From this we can show $\mathfrak{D}$ is associative. By orthogonality we need only prove $[x, y, z]=0$ for $x \in \mathfrak{D}_{i j}, y \in \mathfrak{D}_{j k}, z \in \mathfrak{D}_{k l}$. If the indices are not all distinct we can find $m \neq i, j, k, l$, since $n \geqq 4$, and then $x, y, z \in \mathfrak{D}^{(m)}$; but $D^{(m)}$ is associative since $\mathbb{C}^{(m)}$ is, and we have $[x, y, z]=0$. If the indices are distinct, we have $x=\delta_{i j}(a), y=\delta_{j k}(b), z=\delta_{k l}(c)$ for $a \in \mathfrak{A}_{i j}, b \in \mathfrak{A}_{j k}$, $\iota \in \mathfrak{A}_{k l}$ by (1.1), and

$$
\begin{aligned}
4[x, y, z] & =4\left\{\delta_{i j}(a) \delta_{j k}(b)\right\} \delta_{k l}(c)-4 \delta_{i j}(a)\left\{\delta_{j k}(b) \delta_{k l}(c)\right\} \\
& =2 \delta_{i k}(a \cdot b) \delta_{k l}(c)-2 \delta_{i j}(a) \delta_{j l}(b \cdot c) \\
& =\delta_{i l}((a \cdot b) \cdot c)-\delta_{i l}(a \cdot(b \cdot c))=0
\end{aligned}
$$

by (1.4) and the Peirce relation $[a, b, c]=0$ in $\mathfrak{A}$. Thus $\mathfrak{D}$ is associative. 
The isomorphism of $\mathfrak{S}^{(k)}$ with $\mathfrak{D}^{(k)}$ also furnishes us with specializations $\delta^{(k)}$ of $\mathfrak{A}^{(k)}$ in $\mathfrak{D}^{(k)}$ by $\mathfrak{A}^{(k)} \rightarrow \mathfrak{C}^{(k)} \rightarrow \mathbb{I}^{(k)}$. We can piece these together to give a map $\delta: \mathfrak{A} \rightarrow \mathfrak{D}$ because $\delta^{(k)}=\delta^{(l)}$ on $\mathfrak{A}_{i j} \subset \mathfrak{A}^{(k)} \cap \mathfrak{A}^{(l)}$ for $i, j \neq k, l$. Indeed, since $\delta_{i j}^{(k)}=\phi_{i j}^{(k)} \gamma_{i j}^{(k)}$ this follows from (3) if $i \neq j$. For $i=j$ it suffices to prove $\delta^{(k)}=\delta^{(l)}$ on $a \in \mathfrak{A}_{i i}$ of the form $a=P_{i i}\left(b^{2}\right)$ for $b \in \mathfrak{A}_{i m}$ by (I) (where we can choose $m \neq i, k, l$ since $n \geqq 4$ ); but $\gamma^{(k)}(a)=P_{i i} \gamma^{(k)}\left(b^{2}\right)=P_{i i} \gamma^{(k)}(b) \gamma^{(k)}(b)=\gamma_{i i m}^{(k)}(b \otimes b)$ by $(1.3)$, so $\delta^{(k)}(a)$ $=\phi_{i i}^{(k)} \gamma_{i i m}^{(k)}(b \otimes b)$, and similarly for $\delta^{(l)}(a)$, so the result follows from (3) again.

The map $\delta$ so obtained is a specialization of $\mathfrak{A}$ in $\mathfrak{I}$. It is enough to check the multiplicative property

$$
\delta(a \cdot b)=\frac{1}{2}\{\delta(a) \delta(b)+\delta(b) \delta(a)\} \quad \text { for } a \in \mathfrak{A}_{i j}, b \in \mathfrak{A}_{j k}
$$

by orthogonality. But then $a, b \in \mathfrak{A}^{(l)}$ for $l \neq i, j, k$ and the multiplicative property holds because $\delta$ coincides on $\mathfrak{A}^{(l)}$ with the specialization $\delta^{(l)}$. This also shows $\delta$ is injective, since each $\delta^{(l)}$ is injective as the composite of the injective maps $\gamma^{(l)}$ and $\phi^{(l)}$.

Thus we have constructed a specialization $\delta$ of $\mathfrak{A}$ in an associative algebra $\mathfrak{D}$ which is injective, proving $\mathfrak{A}$ is special.

\section{REFERENCES}

1. N. Jacobson, $A$ coordinatization theorem for Jordan algebras, Proc. Nat. Acad. Sci. U.S.A. 48 (1962), 1154-1160.

2. - Structure theory for a class of Jordan algebras, Proc. Nat. Acad. Sci. U.S.A. 55 (1966), 243-251.

3. K. McCrimmon, $A$ general theory of Jordan rings, Proc. Nat. Acad. Sci. U.S.A. 56 (1966), 1072-1079.

4. - Structure and birepresentations of noncommutative Jordan algebras, Trans. Amer. Math. Soc. 121 (1966), 187-199.

5. W. Martindale, Jordan homomorphisms of symmetric elements of a ring with involution, J. Algebra 7 (1967), 1-18.

UNIVERSITY OF VIRGINIA 